\title{
Care Leaver aus Pflegefamilien
}

\author{
Soziale Beziehungen und Zugehörigkeiten im Übergang aus Pflegefamilien \\ ins Erwachsenenleben
}

Der Prozess des „Leaving Care” aus Pflegefamilien in ein eigenverantwortliches Leben gestaltet sich für diese junge Menschen oft herausfordernd. Soziale Beziehungen haben dabei eine besondere Bedeutung für eine gelingende Übergangsgestaltung. Im Folgenden wird dargestellt, wer insbesondere unterstützend im Prozess des Leaving Care war und wie dabei (familiäre) Zugehörigkeiten "konstruiert" werden.

$\mathrm{O}$ bwohl seit ca. 2012 in Deutschland vermehrt Forschungen durchgeführt werden, die mit dem anglizistischen Label „Care Leaver“1 bzw. „Leaving Care“ versehen sind, gibt es bislang immer noch sehr wenige Erkenntnisse zu Care Leavern aus Pflegefamilien. Insbesondere wurden bisher viel zu selten die jungen Menschen selbst zu ihren Erfahrungen und sozialen Unterstützungsbeziehungen im Übergang

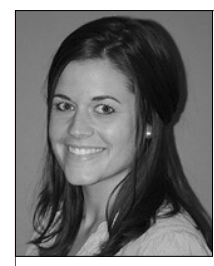

\section{Carolin Ehlke}

Hildesheim, Deutschland

*1987, Dr. phil., Studium der Erziehungswissenschaft an der Universität Hildesheim, seit 2013 wissenschaftliche Mitarbeiterin am Institut für Sozial- und Organisationspädagogik, Universität Hildesheim.

carolin.ehlke@uni-hildesheim.de

Zusammenfassung Seit fast zehn Jahren wird auch in Deutschland intensiver zum Thema „Leaving Care“ und „Care Leaver" geforscht. Trotz dessen haben sich bislang nur sehr wenige Studien mit dem Übergang von jungen Menschen aus Pflegefamilien ins Erwachsenenleben beschäftigt. Zudem wurden nur selten Care Leaver selbst zu ihren Übergangserfahrungen befragt. Daher ist das Wissen darum, wie junge Menschen aus Pflegefamilien den Prozess des Leaving Care aus ihrer Sicht erleben, noch rar. Die Studie „Care Leaver aus Pflegefamilien“ der Autorin hat sich dieser Forschungslücken angenommen und u. a. untersucht, wie und von wem die jungen Menschen während des Übergangs unterstützt wurden. Im Fokus des Beitrags stehen somit soziale Beziehungen sowie die darin deutlich werdende Konstruktion von (familiären) Zugehörigkeiten. Zusammenfassend wird vorgeschlagen, unter dem Konzept eines „doing relationships“ viel stärker die unterschiedlichen Beziehungen von Care Leavern im Hilfe- bzw. Übergangsprozess in den Blick zu nehmen.

Schlüsselwörter Care Leaver, Pflegefamilien, Pflegekinderhilfe, Vollzeitpflege, Soziale Unterstützung, Zugehörigkeit(en), Familialität, Doing Relationships aus ihren Pflegefamilien ins Erwachsenenleben befragt (vgl. Reimer und Petri 2017)².

\section{Verlässliche soziale Beziehungen als Schlüssel- ressource}

Empirische Untersuchungen verdeutlichen, dass verlässliche und kontinuierliche Beziehungen eine Schlüsselrolle für die Bewältigung des Übergangs aus den Erziehungshilfen in ein eigenverantwortliches Leben spielen (vgl. u. a. Rosenbauer 2011; Diakonie Rheinland-Westfalen-Lippe e. V. 2012). Gerade für Care Leaver scheinen sie eine große Bedeutung zu haben. Dies kann an drei Punkten festgemacht werden.

1. Care Leaver erleben, wie andere junge Menschen in ihrem Alter auch, die Herausforderungen des Erwachsenwerdens. Unter der Bezeichnung der „Entgrenzung von Jugend“ (Schröer 2004) ist das sogenannte junge Erwachsensein vor allem geprägt durch Offenheit, Wahlfreiheit und individuelle Gestaltungsspielräume einerseits und Ungewissheit und Unsicherheiten andererseits.

2. Zusätzlich zu diesen Herausforderungen müssen Care Leaver die Umstände bewältigen, dass Erziehungshilfen - Pflegefamilien und Wohngruppen etc. - nicht selten mit Erreichen der Volljährigkeit enden. Dies scheint nach wie vor erstaunlich, da Hilfen gemäß $\$ 41$ SGB VIII bis zum 21. Lebensjahr und in Ausnahmefällen auch noch darüber hinaus gewährt werden sollen. Zudem erhalten viele Gleichaltrige im Vergleich noch viel länger Unterstützung von ihren Eltern, da sie gegenwärtig im Durchschnitt bis zu ihrem 25. Lebensjahr bei ihnen im Elternhaus leben (vgl. BMFSFJ 2017, S. 179). Care Leaver erleben dadurch doppelte Übergangsherausforderungen und sind ,in besonderem Maße den widersprüchlichen Anforderungen des Er- 


\section{Extrablick: Aktuelle Entwicklungen in der Pflegekinderhilfe}

wachsenwerdens ausgesetzt" (Stauber und Walther 2016, S. 154).

3. Dies spitzt sich letztendlich darin zu, dass Care Leaver beispielhaft für junge Menschen in prekären Lebenslagen stehen, die kaum bis keine soziale Unterstützung aus familiären und anderen sozialen Netzwerken erhalten, obwohl sie aufgrund der eigenen Lebensgeschichte besondere Herausforderungen im Leben bewältigen müssen.

Aufgrund dessen ist eigentlich anzunehmen, dass Erziehungshilfen - sowohl während der Hilfen als auch im Leaving Care - den Aufbau und den Erhalt stabiler sozialer Beziehungen fördern. „Dem Fokus auf den Beziehungsaufbau zu Beginn einer Hilfe steht [jedoch] kein vergleichbarer fachlicher Diskurs über die Situation des Hilfeendes und die Gestaltung von Ablöseprozessen aus pädagogischen Beziehungen [...] gegenüber“" (Thomas 2017, S. 147). Im Rahmen der Übergangsvorbereitung wird vielmehr darauf geachtet, dass die jungen Menschen alltagspraktische Fähigkeiten erlernen, um nach dem Ende der Hilfen in der eigenen Wohnung leben zu können. Der Förderung von stabilen sozialen Netzwerken in und außerhalb der Erziehungshilfen wird oft weniger Aufmerksamkeit geschenkt (vgl. ebd., S. 148).

Mit Blick auf Vollzeitpflegeverhältnisse gestaltet sich der Aufbau sozialer Beziehungen zum Teil anders als bei jungen Menschen, die in Wohngruppen aufgewachsen sind. Dies liegt vor allem daran, dass Pflegefamilien Familien sind und Care Leaver zumeist von klein auf bei ihnen gelebt haben. Dies wird im Folgenden differenzierter dargestellt.

\section{Soziale Unterstützung von Care Leavern aus Pflegefamilien}

Care Leaver aus Pflegefamilien erleben verlässliche und kontinuierliche Beziehungen zu ihren Pflegefamilien, zu Freund_innen, zu Partner_innen oder auch zu Vorgesetzten in ihren Ausbildungen und in nebenberuflichen Tätigkeiten als unterstützend im Übergang. Ein durch solche Beziehungen bestehendes soziales Unterstützungsnetzwerk kann sowohl strukturelle Herausforderungen des Übergangs, wie z. B. finanzielle und wohnungsbezogene Notsituationen, abfedern bzw. diesen präventiv entgegenwirken. So berichteten die interviewten Care Leaver mit Blick auf ihre Pflegeeltern, dass sie für sie Extrakonten für finanzielle Rücklagen angelegt haben und einige der jungen Menschen nach der offiziellen Beendigung der Erziehungshilfen in eine eigene Mietwohnung im Haus der Pflegeeltern einziehen konnten.
Andererseits kristallisierte sich auch eine emotionale Unterstützung in Form von Zuwendung und dem Erfahren von Zugehörigkeiten heraus. Die von den interviewten Care Leavern als am positivsten geschilderten Beziehungskonstellationen zeigen dabei das Merkmal der reziproken Wahrnehmung und Vermittlung von Zugehörigkeit(en) auf. Dies bedeutet, dass sowohl die jungen Menschen Zugehörigkeiten vermittelt und ebenso von den jeweiligen Personen(gruppen) Zugehörigkeit erfahren haben. Zugehörigkeiten können dabei sowohl auf einer formal-rechtlichen als auch auf einer emotionalen Ebene verortet werden. Insbesondere emotionale Zugehörigkeiten werden dabei im Prozess des Leaving Care als unterstützend beschrieben (vgl. Ehlke 2019, S. 238 ff.).

\section{Spannungsverhältnisse in den Zugehörigkeits- konstruktionen}

Das Aufwachsen von Care Leavern in bzw. mit mehr als einem Familiensystem zeigt jedoch Spannungen in den Zugehörigkeitskonstruktionen der jungen Menschen auf, die zudem durch das Ende der stationären Hilfen verstärkt werden. Während hinsichtlich der „Herkunftsfamilien“ die formal-rechtliche Zugehörigkeit scheinbar nach außen hin auch über die Volljährigkeit hinaus für andere Personen nachvollziehbar ist, scheint es mit Blick auf Pflegefamilienverhältnisse insbesondere wenn sie mit Ende der stationären Erziehungshilfen formal enden - immer wieder einen Erklärungs- und Rechtfertigungsbedarf zu geben.

Von den befragten Care Leavern wurden Momente beschrieben, in denen sowohl von ihnen selbst als auch von anderen Personen hinterfragt wurde, ob die Pflegefamilien ,richtige“ bzw. „normale Familien" (Care Leaver, 21 Jahre) seien. Daran anknüpfend zeigte sich, dass in der Mehrheit eine emotionale Verbindung zu den jeweiligen Pflegefamilien aufgebaut wurde, die Beziehung zu ihnen als auch der eigene „Pflegekindstatus“ in verschiedenen Kontexten und Alltagssituationen jedoch immer wieder einer Legitimierung bedurften - da die rechtliche Zugehörigkeit eben fehlt. Um den Kreis einer formal-rechtlichen Zugehörigkeit zu schließen, äußerte eine junge Frau im Interview mehrfach den Wunsch nach einer Adoption durch ihre Pflegeeltern. Gleichzeitig wurde aber auch deutlich, dass trotz der formalen Beendigung der Beziehung zu den Pflegefamilien durch das Hilfeende eine emotionale Verbundenheit bestehen blieb, denn die Emotionalität zwischen den Care Leavern und ihren Pflegefamilien kann durch ein formales Hilfeende nicht einfach beendet werden.

Gleichzeitig zeigen sich ambivalente Beziehungskonstellationen zu den „Herkunftsfamilien“. An dieser Stel- 
le konnte ein Spannungsverhältnis zwischen Zugehörigkeitsnegierungen seitens der jungen Menschen bzw. Abgrenzungen von den Familien einerseits und Verantwortlichkeiten ihnen gegenüber und daraus folgende Loyalitätskonflikte andererseits herausgearbeitet werden. Renate Höfer und Kolleg_innen (2017, S. 215) sprechen an dieser Stelle auch von einer Parallelität von Desintegrations- und Integrationsprozessen.

Diesen Ausführungen folgend wird deutlich, dass formal-rechtliche Begrenzungen sowie Verpflichtungen in den Zugehörigkeiten emotionale Zugehörigkeiten - insbesondere im Übergang aus den Pflegefamilien in ein eigenverantwortliches Leben - überlagern und mitunter belasten können. Anders scheint es gelagert zu sein, wenn Care Leaver in Verwandtenpflegeverhältnissen aufgewachsen sind. Zugehörigkeiten im Rahmen von Verwandtenpflegefamilien müssen offensichtlich nicht bzw. nur gering nach außen hin legitimiert werden, denn es ist "quasi noch dieselbe Familie" (Care Leaver, 20 Jahre). Diesbezüglich scheinen die formal-rechtliche als auch die emotionale Zugehörigkeit besser in ein Passungsverhältnis gebracht werden zu können.

\section{„Doing Relationships” im Leaving Care}

Es kann festgehalten werden, dass im Rahmen der Übergangsgestaltung und -begleitung von Care Leavern aus Pflegefamilien die verschiedenen Beziehungskonstellationen und Zugehörigkeitskonstruktionen durch Fachkräfte der Kinder- und Jugendhilfe, aber auch durch andere für die jungen Menschen relevante Unterstützungssysteme, differenziert in den Blick genommen werden müssen. Darauf bezugnehmend sind zwei Aspekte zentral:

- Einerseits gilt es, vor allem die als positiv und unterstützend durch die jungen Menschen wahrgenommenen Beziehungen zu fördern und in die Übergangsgestaltung mit einzubinden - solange dies die jungen Menschen auch möchten. Dies schließt an Erkenntnisse von Andreas Herz (2014, S. 67) an, der konstatiert, dass soziale Beziehungen nicht per se unterstützend sind, sondern ebenso belastend sein können. Belastende Beziehungskonstellationen sollten im Übergang begleitet werden.

- Andererseits sollte sich hinsichtlich der Betrachtung von sozialen Beziehungen auf ein weites Verständnis von familiären bzw. sozialen Netzwerken der jungen Menschen bezogen werden. In Anlehnung an den Ansatz des „doing family“ (vgl. u. a. Schier und Jurczyk 2007) als gemeinsame Konstruktionsleistung von Familie könnte bspw. geschaut werden, wen die jungen Menschen selbst zu ihrem familiären Unterstützungs- netzwerk zählen. So wurde deutlich, dass auch nichtverwandtschaftliche Beziehungen, z. B. zu Vorgesetzten, als familiär beschrieben werden. Eine interviewte junge Frau äußert dazu: „bei meinem Chef und meiner Chefin muss man das so verstehen das ist wie eine Familie also DAS sind eigentlich sagen wir mal so Mutti und Vati" (Care Leaver, 19 Jahre).

Das Konzept „doing family“ muss an dieser Stelle jedoch auch einer kritischen Betrachtung unterzogen werden, da das darin implizierte Verständnis von Familie für die Rekonstruktion bedeutsamer Beziehungen viel zu begrenzt erscheint, mitunter wichtige Beziehungen gar nicht erst in den Blick nimmt. Gerade im Kontext des Aufwachsens in öffentlicher Erziehung bedarf es daher eines reflexiven Hinterfragens des in der Gesellschaft stark positiv besetzten Familienbegriffs.

Daher wäre zu überlegen, dieses Konzept dahingehend zu erweitern, als dass von „doing relationships“ gesprochen wird. Darunter werden generell Netzwerke verstanden (dies können auch familiäre Netzwerke sein), die um verlässliche Fürsorgebeziehungen zentriert sind, jedoch nicht zwangsläufig einer formalen Grundlage bedürfen. Florian Eßer und Stefan Köngeter (2012, S. 39) konstatieren diesbezüglich, dass es in solchen sozialen Netzwerken weniger um rollenförmige Mitgliedschaften geht als vielmehr um die Einbindung in soziale Beziehungen und Rituale oder eben um die erfahrene soziale Unterstützung. Deshalb können auch andere Personen(gruppen), wie z. B. Freund_innen und Partner_innen oder aber auch Tiere für Care Leaver als unterstützend im Übergang aus den Pflegefamilien sein. Die folgenden Aussagen von zwei interviewten Care Leavern untermalen dies:

- Freund_innen: „obne ibn [einen Freund; Anm. d. Verf] [...] hätte ich jede Trennung nicht verkraftet also das ist schon schön so'n so'n Menschen braucht man auch im Leben" (Care Leaver, 19 Jahre)

- Partner_innen: „er [ibr Partner; Anm.d. Verf.] ist halt so mein Rückhalt er ist immer für mich da er war auch da als ich ganz viel Stress mit meiner Mutter hatte und dann hat er mich auch also da hab ich ihm auch mal erzählt ,ja ich kann grad nicht mehr ich heul hier grad nur rum' und dann hat er gesagt, ok ich hol dich von $z u$ Hause ab und nehm dich mit zu mir' so so ist er halt" (Care Leaver, 18 Jahre).

- Tier(e): „mein Pferd ist mein Leben mein Ein ich brauch das einfach wenn ich die nicht hab denn würd ich hier auch so eingehen wie eine Tomate manchmal ((lacht)) also Mimmi mein Pferd die hält schon viel zu mir brauch ich auch" (Care Leaver, 19 Jahre) 


\section{Extrablick: Aktuelle Entwicklungen in der Pflegekinderhilfe}

Diese sozialen Beziehungen von Care Leavern zu Freund_innen, Partner_innen, anderen relevanten Personen oder eben auch zu Tieren gilt es daher sowohl während der Zeit in den Pflegefamilien als auch im Übergang in ein eigenverantwortliches Leben zu stärken. Auch in wissenschaftlichen Forschungen sollten sie mehr Beachtung erhalten.

\section{Soziale Beziehungen fördern: eine Aufgabe der Erziehungshilfen}

Soziale Beziehungen von Care Leavern aus Pflegefamilien mehr in den Blick zu nehmen und sie in der Übergangsbegleitung zu stärken, erscheint ein wichtiger Ansatz zu sein. Dabei darf sich die Kinder- und Jugendhilfe im Prozess des Leaving Care nicht gänzlich auf private bzw. informelle Hilfebeziehungen verlassen. Am Beispiel der Pflegeeltern zeigt sich, dass sie sich aufgrund ihres persönlichen Willens auch nach Beendigung der Erziehungshilfen weiterhin häufig ehrenamtlich für die jungen Menschen zuständig fühlen - ohne dass sie weiter gefördert werden. Sie erhalten zumeist keine Anerkennung, z. B. in Form von einer finanziellen (oder anderen) Aufwandsentschädigung, auch wenn sie eigentlich die Aufgaben übernehmen, für die die Kinder- und Jugendhilfe zuständig ist (vgl. Ehlke 2019, S. 270 f.).

1. Care Leaver sind junge Menschen, die für eine bestimmte Zeit in stationären Erziehungshilfen, z. B. Wohngruppen, Pflegefamilien, Erziehungsstellen oder anderen Betreuungsformen, aufgewachsen sind und von dort aus den Übergang ins Erwachsensein beginnen oder die Hilfesettings bereits verlassen haben (vgl. Ehlke 2019, S. 2). In diesem Beitrag liegt der Fokus auf jenen Care Leavern, die volljährig sind und erst seit wenigen Monaten bis wenigen Jahren keine Erziehungshilfen mehr erhalten.

2. Der Beitrag basiert auf einer Studie (Ehlke 2019), in der sieben Care Leaver im Alter von 18 bis 24 Jahren interviewt und $u$. a. befragt wurden, wie und von wem sie soziale Unterstützung (Nestmann und Wehner 2008; Weinhold und Nestmann 2012) in dieser Zeit erfabren haben.

Funding. Open Access funding provided by Projekt DEAL.

Open Access. Dieser Artikel wird unter der Creative Commons Namensnennung 4.0 International Lizenz veröffentlicht, welche die Nutzung, Vervielfältigung, Bearbeitung, Verbreitung und Wiedergabe in jeglichem Medium und Format erlaubt, sofern Sie den/die ursprünglichen Autor(en) und die Quelle ordnungsgemäß nennen, einen Link zur Creative Commons Lizenz beifügen und angeben, ob Änderungen vorgenommen wurden.

Die in diesem Artikel enthaltenen Bilder und sonstiges Drittmaterial unterliegen ebenfalls der genannten Creative Commons Lizenz, sofern sich aus der Abbildungslegende nichts anderes ergibt. Sofern das betreffende Material nicht unter der genannten Creative Commons Lizenz steht und die betreffende Handlung nicht nach gesetzlichen Vorschriften erlaubt ist, ist für die oben aufgeführten Weiterverwendungen des Materials die Einwilligung des jeweiligen Rechteinhabers einzuholen.

Weitere Details zur Lizenz entnehmen Sie bitte der Lizenzinformation auf http://creativecommons.org/licenses/by/4.0/deed.de.

\section{Literatur}

Bundesministerium für Familien, Senioren, Frauen, Jugendliche (BMFSFJ) (2017). 15. Kinder- und Jugendbericht. Bericht über die Lebenssituation junger Menschen und die Leistungen der Kinder- und Jugendhilfe in Deutschland. Berlin: BMFSFJ.

Diakonie Rheinland-Westfalen-Lippe e. V. (2012). Übergänge in die Zeit nach dem Heim. Ergebnisse aus einem Projekt mit ehemaligen Jugendlichen aus den Erziehungshilfen

Ehlke, C. (2019). Care Leaver aus Pflegefamilien. Die Bewältigung des Übergangs aus der Vollzeitpflege in ein eigenverantwortliches Leben aus Sicht der jungen Menschen. Dissertation. Hildesheim: Universität Hildesheim.

Eßer, F., \& Köngeter, S. (2012). Doing Family in der Heimerziehung. Familialität als professionelle Deutungsressource. Sozial Extra, 36(7/8), S. 3740. https://doi.org/10.1007/s12054-012-0084-9.

Herz, A. (2014). Strukturen transnationaler sozialer Unterstützung. Wiesbaden: Springer.

Höfer, R., et al. (2017). Verwirklichungschance SOS-Kinderdorf. Handlungsbefähigung und Wege in die Selbstständigkeit. Opladen/Berlin/Toronto: Barbara Budrich.

Nestmann, F., \& Wehner, K. (2008). Soziale Netzwerke von Kindern und Jugendlichen. In F. Nestmann, et al. (Hrsg.), Kindernetzwerke. Soziale Beziehungen und soziale Unterstützung in Familie, Pflegefamilie und Heim (S. 11-40). Tübingen: dgvt.

Reimer, D., \& Petri, C. (2017). Wie gut entwickeln sich Pflegekinder? Eine Longitudinalsstudie. Siegen: universi - Universitätsverlag.

Rosenbauer, N. (2011). Selbstständigkeit als Ziel?! Jugendliche und junge Volljährige in den Hilfen zur Erziehung. In Sozialpädagogisches Institut des SOS-Kinderdorf e. V. (Hrsg.), Fertig sein mit 18? (S. 64-83). München: Sozialpädagogisches Institut im SOS-Kinderdorf.

Schier, M., \& Jurczyk, K. (2007). Familie als Herstellungsleistung in Zeiten der Entgrenzung. Aus Politik und Zeitgeschichte, S. 10-17. https:// doi.org/10.1007/978-3-658-15005-1_7.

Schröer, W. (2004). Befreiung aus dem Moratorium? Zur Entgrenzung von Jugend. In K. Lenz, W. Schefold \& W. Schröer (Hrsg.), Entgrenzte Lebensbewältigung. Jugend, Geschlecht und Jugendhilfe (S. 19-74). Weinheim, Basel: Beltz Juventa.

Stauber, B., \& Walther, A. (2016). Junge Erwachsene. Eine exemplarische Lebenslage für die Ausformulierung einer Sozialpädagogik des Übergangs. In W. Schröer, N. Struck \& M. Wolff (Hrsg.), Handbuch Kinder- und Jugendhilfe (S. 135-163). Weinheim, Basel: Beltz Juventa.

Thomas, S. (2017). ...und dann ist Schluss? Zur Transformation sozialer Beziehungen im Übergang von stationären Erziehungshilfen ins Erwachsenenleben. Forum Erziehungshilfen, 23(3), S. 147-150.

Weinhold, K. \& Nestmann, F. (2012). Soziale Netzwerke und soziale Unterstützung in Übergängen. In S. B. Gahleitner \& G. Hahn (Hrsg.), Übergänge gestalten, Lebenskrisen begleiten (2. Aufl. S. 52-67). Bonn: Psychiatrie-Verlag. 